\title{
Parametric Myocardial Perfusion PET Imaging using Physiological Clustering
}

\author{
Hassan Mohy-ud-Din ${ }^{a, b, *}$, Nikolaos A. Karakatsanis, $\mathrm{PhD}^{b}$, Martin A. Lodge, $\mathrm{PhD}^{b}$, \\ Jing Tang, $\mathrm{PhD}^{c}$, and Arman Rahmim, $\mathrm{PhD}^{a, b}$ \\ ${ }^{a}$ Department of Electrical and Computer Engineering, Johns Hopkins University, USA; \\ ${ }^{b}$ Department of Radiology and Radiological Sciences, Johns Hopkins University, USA; \\ ${ }^{c}$ Department of Electrical and Computer Engineering, Oakland University, USA
}

\begin{abstract}
We propose a novel framework of robust kinetic parameter estimation applied to absolute flow quantification in dynamic PET imaging. Kinetic parameter estimation is formulated as a nonlinear least squares with spatial constraints problem (NLLS-SC) where the spatial constraints are computed from a physiologically driven clustering of dynamic images, and used to reduce noise contamination. An ideal clustering of dynamic images depends on the underlying physiology of functional regions, and in turn, physiological processes are quantified by kinetic parameter estimation.

Physiologically driven clustering of dynamic images is performed using a clustering algorithm (e.g. $K$-means, Spectral Clustering etc) with Kinetic modeling in an iterative handshaking fashion. This gives a map of labels where each functionally homogenous cluster is represented by mean kinetics (cluster centroid). Parametric images are acquired by solving the NLLS-SC problem for each voxel which penalizes spatial variations from its mean kinetics. This substantially reduces noise in the estimation process for each voxel by utilizing kinetic information from physiologically similar voxels (cluster members). Resolution degradation is also substantially minimized as no spatial smoothing between heterogeneous functional regions is performed.

The proposed framework is shown to improve the quantitative accuracy of Myocardial Perfusion (MP) PET imaging, and in turn, has the long-term potential to enhance capabilities of MP PET in the detection, staging and management of coronary artery disease.
\end{abstract}

Keywords: myocardial perfusion, coronary flow reserve, coronary artery stenosis, coronary artery disease, PET, $K$-means clustering, spectral clustering, physiological clustering, penalized least squares

\section{INTRODUCTION}

Several studies worldwide have attributed a high "morbidity" and "mortality" rate to cardiovascular diseases. ${ }^{1-5}$ The World Health Organization has predicted that by 2030 approximately 23.6 million people will die due to Coronary Artery Diseases (CAD). This is a staggering increase of $\sim 36.4 \%$ since 2008. ${ }^{6}$ The importance of this matter can also be gauged from the fact that the 2013 issue of "Atlas of Nuclear Cardiology" is fully devoted to the instrumentation, experimentation, assessment, and analysis of biomarkers for the early detection, staging and management of CAD. ${ }^{7}$

Absolute quantification of myocardial blood flow $(\mathrm{MBF})$ and coronary flow reserve $\left(\mathrm{CFR}=\frac{M B F \text { at peak-stress }}{M B F \text { at peak-rest }}\right)$ has provided new insights over conventional myocardial perfusion imaging (MPI) by allowing early detection of preclinical atherosclerosis and providing an opportunity to modify risk factors or initiate treatment. ${ }^{5,8,9} \mathrm{CFR}$ has been shown to be related to the degree of coronary artery stenosis (CAS). ${ }^{10}$ It thus allows for noninvasive assessment of the functional importance of CAS and may aid identification of patients with either diffuse, nonocclusive luminal coronary artery narrowing or a balanced reduction in coronary artery blood flow (extensive multi-vessel coronary disease). ${ }^{11}$

*For further information please contact Hassan Mohy-ud-Din, email: hmohyud1@jhu.edu

Medical Imaging 2014: Biomedical Applications in Molecular, Structural, and Functional Imaging,

edited by Robert C. Molthen, John B. Weaver, Proc. of SPIE Vol. 9038, 90380P

(C) 2014 SPIE · CCC code: 1605-7422/14/\$18 - doi: 10.1117/12.2043947

Proc. of SPIE Vol. 9038 90380P-1 
Positron Emission Tomography (PET) is a powerful imaging modality that enables noninvasive and accurate quantification of MBF and CFR by administration of a radio-pharmaceutical tagged with positron emitter. ${ }^{7,12-14}$ PET, as compared to SPECT, has high sensitivity, superior spatio-temporal resolution, and accurate attenuation correction. ${ }^{8,15-19}$

Despite all these pearls, absolute quantification of MBF and CFR has hardly translated into clinical practice and, thus far, remains a research adventure. A major impediment is the production and delivery of short-lived radio-tracers at the clinical site. ${ }^{82} R b$ is one of the most commonly used radio-tracer for absolute quantification of MBF and CFR. ${ }^{20-22}$ It does not require on-site cyclotron for production and has a short half-life of 76 secs enabling fast serial imaging $\left(\sim 15 \mathrm{~min}^{23}\right)$ and high patient throughput. ${ }^{82} R b$ dynamic PET images suffer from high noise-levels which adversely impacts accurate quantification of MBF and CFR especially at the voxel-level (parametric images).

One way to address the issue of increased noise-levels (poor SNR) is to perform heavy post-smoothing $(F W H M=5-12 \mathrm{~mm})$ on reconstructed dynamic images before extracting parametric images by kinetic modeling. ${ }^{24}$ This comes at a cost of resolution degradation (blurring) due to smoothing operator. Another approach is to perform ROI quantification of MBF and CFR where the voxel intensities are averaged over each region-of-interest (ROI). ${ }^{25}$ This also comes at a cost of loss of spatial information and underlying tissue heterogeneity.

We propose a novel framework of robust kinetic parameter estimation at the individual voxel level that substantially reduces noise using "Physiological Clustering". Physiological clustering is an approach of clustering a PET image driven by the underlying physiology. The resulting label map (image) is a union of disjoint clusters each represented by its mean kinetics. The parameter estimation problem is then formulated as a nonlinear least squares with spatial constraints (NLLS-SC) where the spatial constraints are derived from the physiologically clustered image. Figure 1 shows a flowchart of the proposed approach.

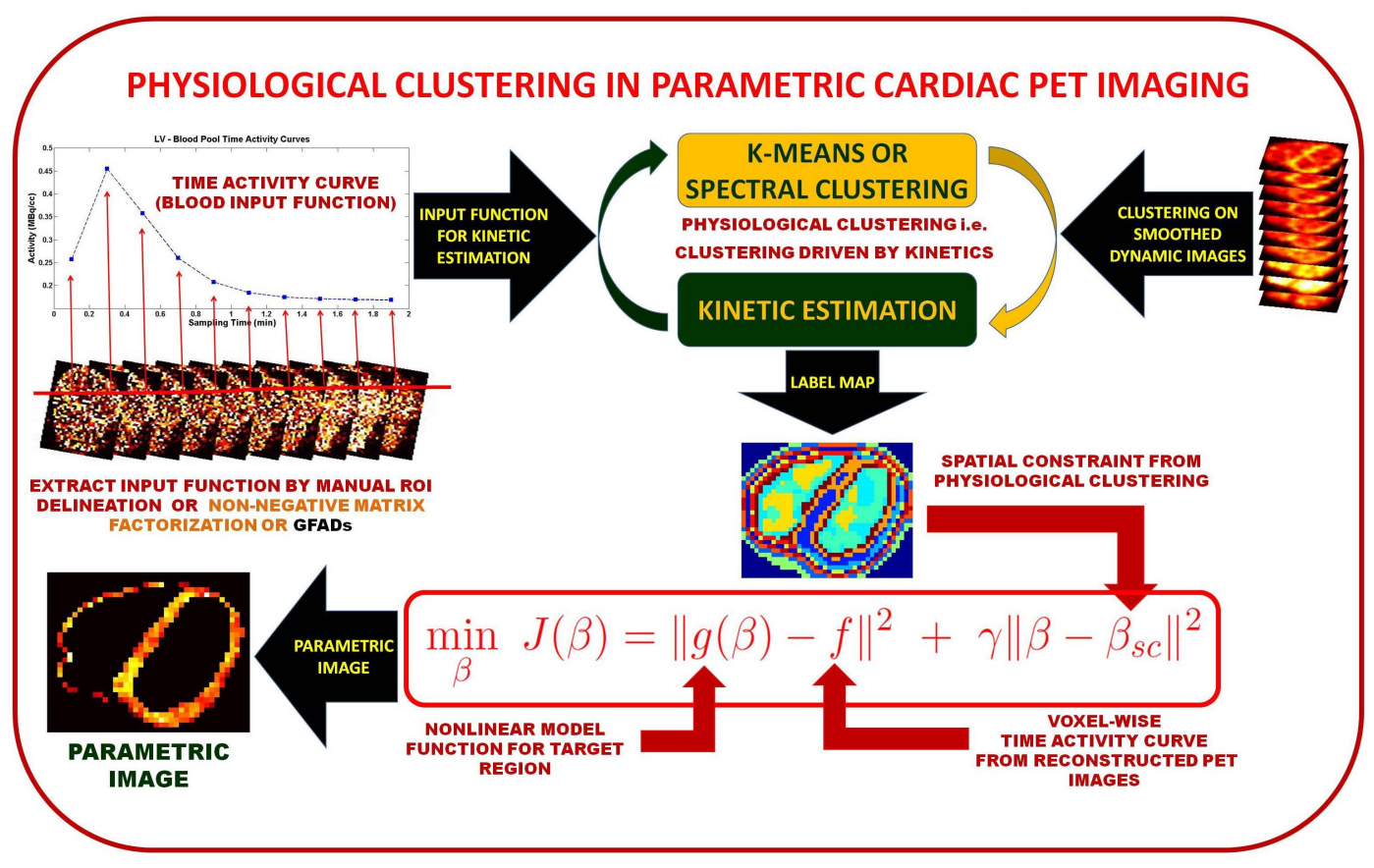

Figure 1. Flowchart of the proposed approach. 


\section{THEORY}

\subsection{Pharmacokinetic Model for ${ }^{82} R b$ radio-tracer}

${ }^{82} R b$ radio-tracer kinetics can be described by a one-tissue compartment model ${ }^{26,27}$ as shown in Figure 2 . The myocardial activity concentration, $C_{m y o}(t)$, is defined as the convolution of arterial blood concentration, $C_{a}(t)$, and model impulse response, $h(t)$ :

$$
C_{m y o}(t)=C_{a}(t) \otimes h(t)
$$

where $h(t)$ is a function of the transport rate constants $K_{1}$ (uptake rate in units of $\mathrm{mL} / \mathrm{min} / \mathrm{g}$ ) and $k_{2}$ (washout rate in units of $1 / \mathrm{min})$ and density of myocardial tissue $\rho(1.04 \mathrm{~g} / \mathrm{mL}):^{28}$

$$
h(t)=\rho K_{1} e^{-k_{2} t}
$$

Due to partial volume effects (PVE), caused by the limited resolution of PET scanners, the measured myocardial tissue concentration, $C_{\text {meas }}(t)$, is contaminated by "spill-over" of activity from arterial blood:

$$
\begin{aligned}
C_{\text {meas }}(t) & =f_{a} C_{a}(t)+\left(1-f_{a}\right) C_{m y o}(t) \\
& =f_{a} C_{a}(t)+\left(1-f_{a}\right) C_{a}(t) \otimes \rho K_{1} e^{-k_{2} t}
\end{aligned}
$$

where $f_{a}$ is the fractional blood volume spillover that accounts for contribution of blood activity in the measured myocardial concentration curve and $\left(1-f_{a}\right)$ corrects for partial volume loss in the myocardium. ${ }^{27,29}$

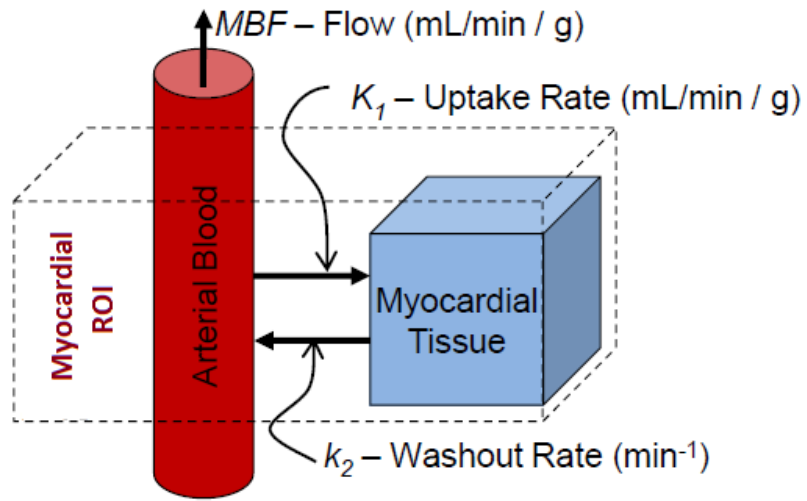

Figure 2. One-tissue Compartment Model for ${ }^{82} R b$ radio-tracer where activity concentration in the myocardial ROI is a convex mixture of concentration curves from arterial blood and myocardial tissue (reproduced from Klein $2010^{28}$ ).

Unlike ${ }^{13} N$-ammonia radio-tracer, where the uptake rate is proportional to MBF, $K_{1}$ estimates from a ${ }^{82} R b$ study needs to be corrected for extraction fraction, $E F$, which is analytically described by the Renkin-Crone model: ${ }^{30,31}$

$$
K_{1}=E F \times M B F=\left(1-a e^{-b / M B F}\right) \times M B F
$$

The extraction fraction, $E F$, is a nonlinear function of MBF and decreases with increase in MBF due to tracer extraction from blood via diffusion and active transport. ${ }^{26,27,30,31}$ Many studies have reported the Rekin-Crone model parameters $(a$, and $b$ ) for various radio-tracers and a comprehensive table can found in Klein et. al. 2010 (Table 4). ${ }^{27}$ In this study $a=0.77$ and $b=0.63 .{ }^{26}$

\subsection{Image Derived Input Function (IDIF)}

Parameter estimation requires knowledge of input function, $C_{a}(t)$ (Equations 1 and 4). In clinical practice, the input function is measured invasively by arterial cannulation which is a cumbersome procedure, both for the clinician and the patient. This calls for alternative ways to acquire input function. IDIF are extremely promising and feasible methods that extract the input function directly from the acquired dynamic images. ${ }^{32}$ In cardiac imaging, this approach is readily applicable due to the presence of large blood pools of Left Ventricle (LV) and Right Atrium (RA) in the PET field of view. ${ }^{8}$ 
Arterial concentration curve, $C_{a}(t)$, is extracted from co-registered dynamic PET images by placing an elliptical ROI $\left(50 \mathrm{~mm}^{2}\right)$ in the LV blood pool. These curves are generated from 4 mid-ventricular imaging planes and then averaged to reduce noise. ${ }^{33}$

Other approaches use Factor Analysis (FA) ${ }^{34}$ or Non-negative Matrix Factorization (NMF) $)^{35,36}$ to extract concentration curves from the LV, RV, and myocardial tissue. The basic essence underlying FA and NMF is the same: the dynamic data set $(A)$ is factorized into a product of factor images $(W)$ and coefficient matrix $(H)$ :

$$
A_{N \times M}=W_{N \times r} H_{r \times M}
$$

where $r$ is the number of pre-defined factors, $N$ is the number of voxels, and $M$ is the number of dynamic frames. The rows of $H$ matrix provide the TACs for the $r$ factors. In myocardial perfusion PET imaging, $r=3$ (RV, $\mathrm{LV}$, and myocardium). A major shortcoming of these splitting techniques is the nonuniqueness of the solution matrices $\left(W\right.$ and $H$ ), upto a rotation matrix $Q$, as shown below: ${ }^{37,38}$

$$
A=\left(W Q^{-1}\right)(Q H)=\tilde{W} \tilde{H}
$$

where $W, \tilde{W}, H$, and $\tilde{H}$ are non-negative matrices. Nonuniqueness in FA is addressed by imposing minimal structure overlap (MSO) constraint as implemented in Generalized Factor Analysis for Dynamic Sequences (GFADs). ${ }^{23,39} \mathrm{NMF}$ is preferred over GFADs which uses conjugate gradient algorithm as opposed to simple multiplicative/additive update equation in $\mathrm{NMF}^{40}$

\subsection{Physiological Clustering}

Clustering techniques for enhanced parameter estimation has been reported before ${ }^{41-45}$ However, in this work clustering is driven by the underlying physiology of functional regions. Physiological clustering approach for noisereduction is based on an explicit statement: accurate kinetic parameter estimation requires a segmentation; ideal segmentation requires knowledge of the underlying physiological parameters. ${ }^{46}$ This dilemma is easily addressed by an iterative "handshaking" algorithm where kinetics drive clustering and clustering drives kinetics.

Algorithm 1 describes the simplest physiological clustering approach that uses $K$-means with Kinetic Modeling $(\mathrm{KM}-\mathrm{KM})^{46}$ to generate a labeling where each label represents a distinct functional region. $K$-means clustering uses the Euclidean norm of Time Activity Curves (TACs) to gauge physiological similarity of pair of voxels indexed by $(i, j)$ :

$$
S_{K \text {-means }}(i, j)=\left\|T A C_{i}-T A C_{j}\right\|_{2}
$$

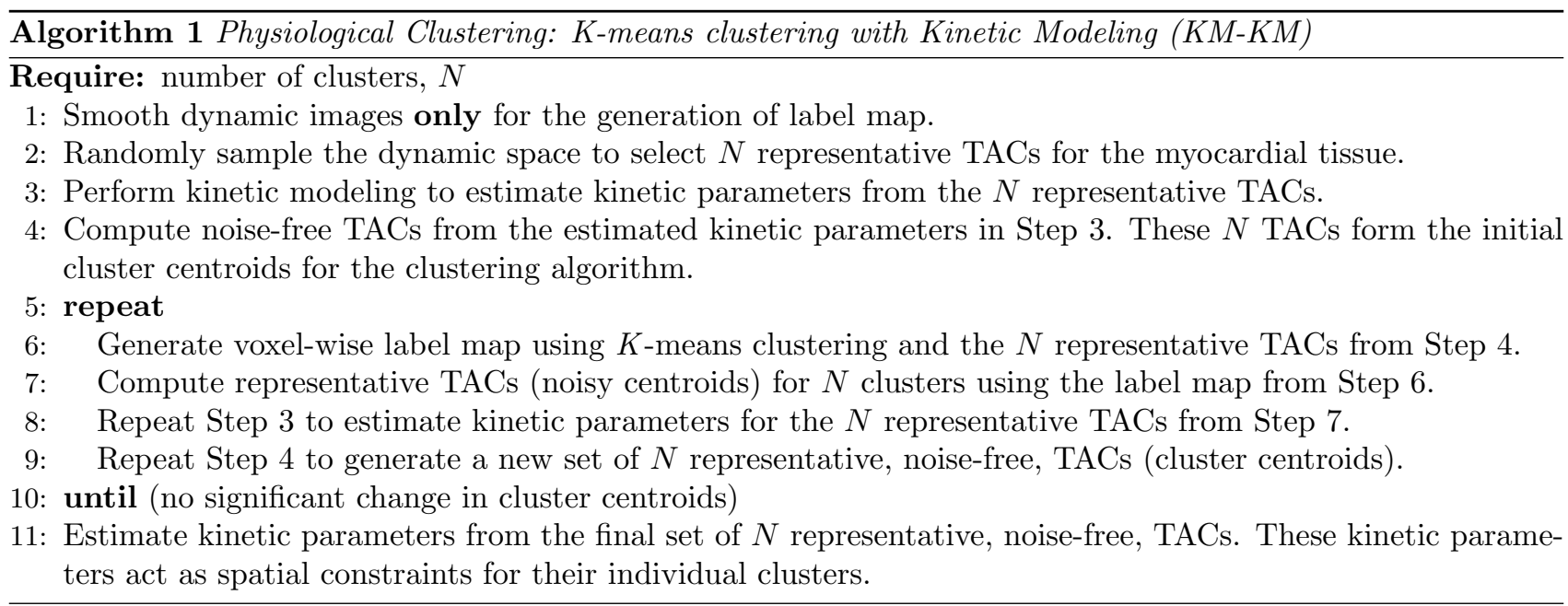

$K$-means clustering, though extremely simple and straightforward, is sensitive to the initialization of representative TACs due to non-convex objective function, requires apriori specification of number of clusters, and 
the similarity metric does not incorporate spatial proximity of voxels. Non-convexity is addressed by convexrelaxation of the $K$-means objective function:

$$
\min _{\boldsymbol{U}} F_{\eta}(\boldsymbol{U})=\frac{1}{2} \sum_{i=1}^{p}\left\|\boldsymbol{x}_{\boldsymbol{i}}-\boldsymbol{u}_{\boldsymbol{i}}\right\|_{2}^{2}+\eta \sum_{i<j} w_{i, j}\left\|\boldsymbol{u}_{\boldsymbol{i}}-\boldsymbol{u}_{\boldsymbol{j}}\right\|
$$

where $p$ is the number of voxels, $\eta$ is a tuning parameter, $w_{i, j}$ is a non-negative weight, $\boldsymbol{x}_{\boldsymbol{i}}$ is the TAC for voxel $i$, and the $i^{t h}$ column of matrix $\boldsymbol{U}$ (i.e. $\boldsymbol{u}_{\boldsymbol{i}}$ ) is the cluster centroid for voxel $i$. An interesting aspect of this convexification is that it obviates the need of predefining number of clusters $(N)$ at the cost of fixing the tuning parameter $\eta$. This problem can be solved using Alternating Direction Method of Multipliers (ADMM). ${ }^{47}$

The similarity metric in Equation 8 can be easily modified to account for spatial proximity of voxels. Such a similarity metric is commonly observed in Normalized Cuts and Spectral Clustering algorithms. ${ }^{4-50}$

$$
S_{\text {Spec. Clust. }}(i, j)=\exp \left(-\frac{\left\|T A C_{i}-T A C_{j}\right\|_{2}^{2}}{\sigma_{T A C}^{2}}\right) \exp \left(-\frac{\left\|X_{i}-X_{j}\right\|_{2}^{2}}{\sigma_{X}^{2}}\right)
$$

for $\left\|X_{i}-X_{j}\right\|_{2}<r$ where $X_{i}$ is the spatial location of voxel $i$.

In a nutshell, we have a nice family of clustering algorithms that generate a label map (image) where each functionally homogeneous region is represented by mean kinetics. These mean kinetics form the spatial constraints set for robust parameter estimation.

\subsection{Robust Parameter Estimation}

Robust estimation of physiologically meaningful parameters (like the uptake rate $K_{1}$ ) is achieved by solving the following nonlinear least squares with spatial constraints (NLLS-SC) optimization problem for each voxel:

$$
\min _{\boldsymbol{\beta}} J(\boldsymbol{\beta})=\left\|\boldsymbol{C}_{\text {meas }}(\boldsymbol{t} ; \boldsymbol{\beta})-\boldsymbol{C}_{\boldsymbol{P E T}}(\boldsymbol{t})\right\|_{2}^{2}+\gamma\left\|\boldsymbol{\beta}-\boldsymbol{\beta}_{\boldsymbol{s c}}\right\|_{2}^{2}
$$

where $\boldsymbol{\beta}=\left[\begin{array}{lll}K_{1} & k_{2} & f_{a}\end{array}\right]^{T}$ is the desired parameter vector, $\boldsymbol{C}_{\text {meas }}(\boldsymbol{t} ; \boldsymbol{\beta})$ models the measured PET signal (Equation 4), $\boldsymbol{C}_{\boldsymbol{P E T} \boldsymbol{T}}(\boldsymbol{t})$ is the measured TAC, and $\boldsymbol{\beta}_{\boldsymbol{s c}}$ is the spatial constraint derived from physiological clustering of dynamic images. Each voxel belongs to one of the $N$ clusters and the representative kinetic (physiological) parameter vector for that cluster forms the spatial constraint vector $\left(\boldsymbol{\beta}_{\boldsymbol{s c}}\right) . \gamma$ penalizes large deviations from mean kinetics. Equation 11 is solved using using a trust-region-reflective nonlinear least-squares algorithm. MBF is estimated from $K_{1}$ by solving Equation 5 using a fixed-point iteration approach. ${ }^{51}$ CFR is computed as the ratio of MBF at peak-stress and peak-rest.

Table 1. Kinetic parameters used in the simulation of cardiac PET images for ${ }^{82} R b$ radio-tracer.

\begin{tabular}{|c|c|c|}
\hline Tissue & $\boldsymbol{K}_{\mathbf{1}}(\mathbf{m L} / \mathbf{m i n} / \mathbf{g})$ & $\boldsymbol{k}_{\mathbf{2}}(\mathbf{1} / \mathbf{m i n})$ \\
\hline Liver & 0.57 & 0.97 \\
\hline Lung & 0.18 & 0.98 \\
\hline Muscle & 0.06 & 0.21 \\
\hline Myocardium (rest) & 0.70 & 0.16 \\
\hline Myocardium (stress) & 1.48 & 0.32 \\
\hline Myocardiym Defect & 0.74 & 0.25 \\
\hline
\end{tabular}

\section{SIMULATION}

We simulated a set of dynamic cardiac PET images acquired in 2-D mode (with septa) using XCAT phantom $\left(128 \times 128 \times 47\right.$ voxels, $3.27 \times 3.27 \times 3.27 \mathrm{~mm}^{3}$ per voxel). TACs were generated for five tissues (left ventricle $(\mathrm{LV})$, right ventricle (RV), myocardium, liver, lung, and muscle) using realistic kinetic parameters and a one-tissue compartment model. Table 1 lists the kinetic parameters for different tissues. Arterial blood fraction for the 

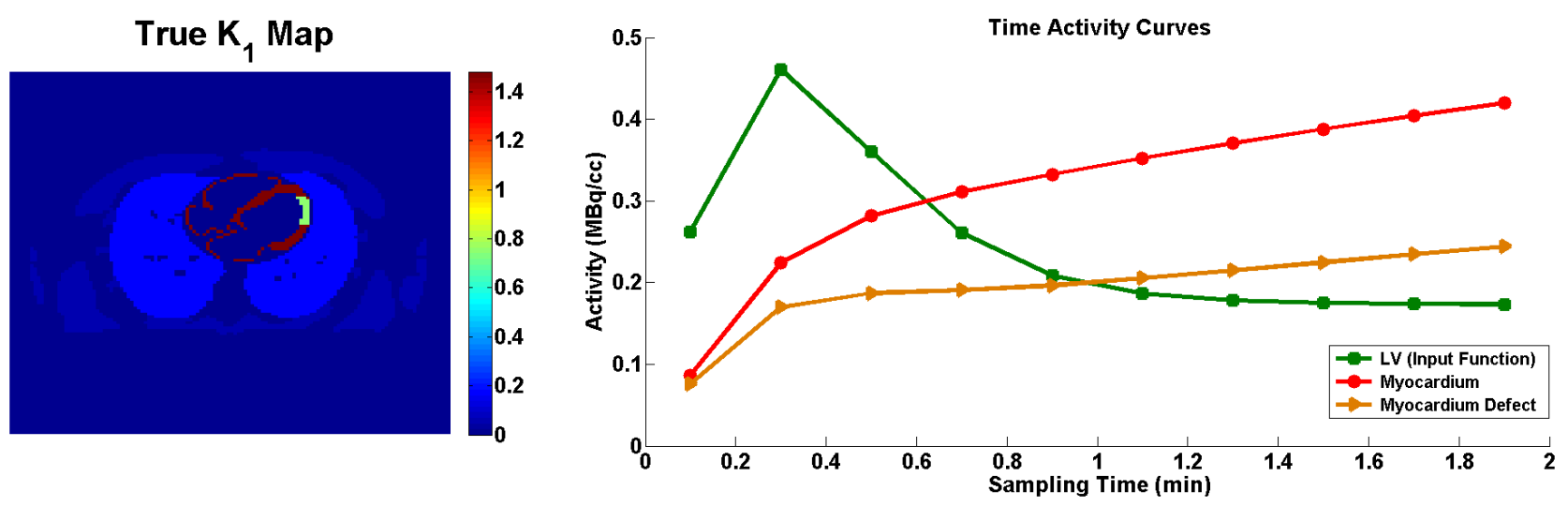

Figure 3. True $K_{1}$ image and the TACs generated using a one-tissue compartment model for ${ }^{82} R b$ radio-tracer.

myocardial tissue was assumed to be $25 \%$. Figure 3 shows the true $K_{1}$ image and the noise-free TACs for LV (input function), normally perfused myocardium and perfusion defect.

The dynamic data set consists of 10 time frames $(10 \times 12$ secs $)$ spanning a total duration of 2 minutes. Dynamic images were forward projected using a precomputed 2-D projection matrix with 315 angular samples over $180^{\circ}$, and 323 radial bins with $2.26 \mathrm{~mm}$ spacing. Positron range blurring, attenuation, normalization, randoms, scatter, and decay were also incorporated. Positron range effect was simulated by blurring the dynamic images with a space-invariant kernel, $h(\boldsymbol{r})=e^{-0.56 r}$, as previously derived in Rahmim et. al. 2008. ${ }^{52}$ For randoms and scatter, a uniform distribution was assumed in the projection space with the randoms and scatter fractions set to be $20 \%$ each. Poisson noise was then added to the dynamic sinograms which resulted in a total number of 10 million events for a 2 minutes study. Noisy sinograms were reconstructed using the OSEM algorithm (2 iterations, and 21 subsets). The input function was extracted using a manually placed elliptical ROI over the LV (Section 2.2). A 2-D Gaussian filter (size $3 \times 3$, standard deviation 0.5) was used to smooth the reconstructed dynamic images for clustering and five clusters were assumed a priori $(N=5)$. Physiological clustering was performed on the smoothed reconstructed dynamic images using Algorithm 1 (Section 2.3). Parametric images were generated using the non-smoothed reconstructed dynamic images (Section 2.4) and the IDIF. 50 noise realizations were generated for rest and stress analysis with varying penalty parameter $\left(\gamma=0-1 \times 10^{-2}\right.$ where $\gamma=0$ corresponds to no spatial constraint).

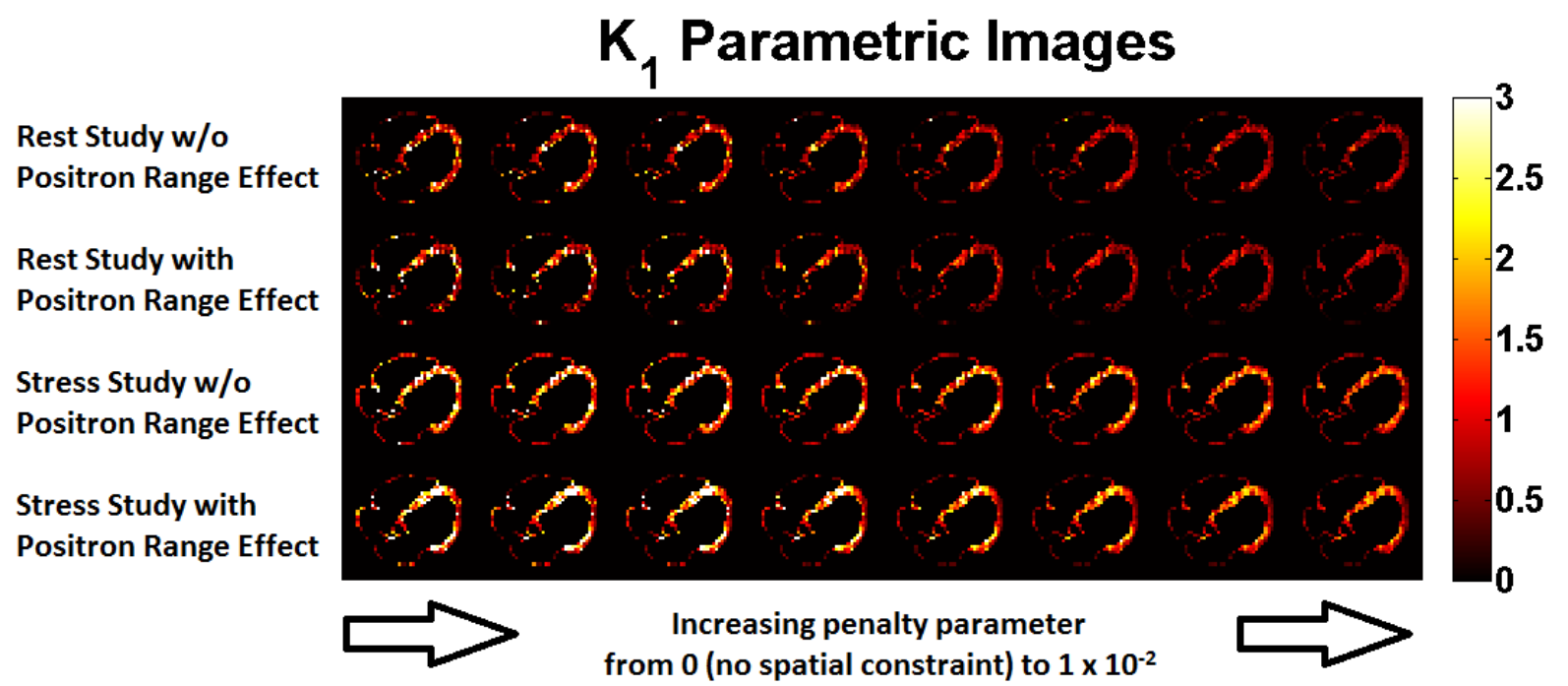

Figure 4. $K_{1}$ parametric images obtained by varying the penalty parameter, $\gamma$, from 0 (no spatial constraint) to $1 \times 10^{-2}$. 
Figure 4 shows parametric images of $K_{1}$, from rest and stress simulations, for different penalty parameters $\left(\gamma=0,5 \times 10^{-5}, 1 \times 10^{-4}, 3 \times 10^{-4}, 9 \times 10^{-4}, 2 \times 10^{-3}, 4 \times 10^{-3}\right.$, and $\left.8 \times 10^{-3}\right)$. We also explored the impact of positron range blurring on parametric image estimation by simulating cardiac PET images with and without positron range blurring. Positron range blurring produced noisier $K_{1}$ images in rest and stress simulations. This may be attributed to the loss of counts in the myocardial tissue due to PVE. Qualitative analysis reveal that as spatial constraint is enforced (by increasing $\gamma$ ) noise in $K_{1}$ images is reduced thereby enhancing image quality.

Figure 5 shows a bias versus variance tradeoff obtained over 50 noise realizations. Different points on the curves are obtained by varying the penalty parameter $\left(\gamma \in\left[0,1 \times 10^{-2}\right]\right)$. Quantitative analysis show that as we impose spatial constraints $(\gamma>0)$ on conventional parameter estimation $(\gamma=0)$ noise and bias decrease with increasing $\gamma$ till a point, $\gamma^{*}$, is reached that gives a minimum $\left[\right.$ noise $\left(\gamma^{*}\right)$, bias $\left.\left(\gamma^{*}\right)\right]$ performance. For $0<\gamma \leq \gamma^{*}$, physiological clustering reduces noise and noise-induced bias in parametric images by exploiting information from functionally (kinetically) similar voxels. We also observe that, for matched noise performance, positron range blurring enforces a stronger penalty on the spatial constraints producing increased estimation bias. For $\gamma>\gamma^{*}$, noise is further reduced at the expense of increased bias since the voxel-wise kinetics in each cluster is smoothed to match mean kinetics. Without positron range blurring, $K_{1}$ rest images showed a $\sim 12 \%$ reduction in noise and $\mathrm{a} \sim 40 \%$ reduction in bias $\left(\gamma^{*}=6 \times 10^{-4}\right)$, and $K_{1}$ stress images showed a $\sim 5 \%$ reduction in noise and a $\sim 16 \%$ reduction in bias $\left(\gamma^{*}=3 \times 10^{-4}\right)$. With positron range blurring, $K_{1}$ rest images showed a $\sim 8 \%$ reduction in noise and a $\sim 34 \%$ reduction in bias $\left(\gamma^{*}=6 \times 10^{-4}\right)$, and $K_{1}$ stress images showed a $\sim 1 \%$ reduction in noise and $\mathrm{a} \sim 6 \%$ reduction in bias $\left(\gamma^{*}=5 \times 10^{-5}\right)$.

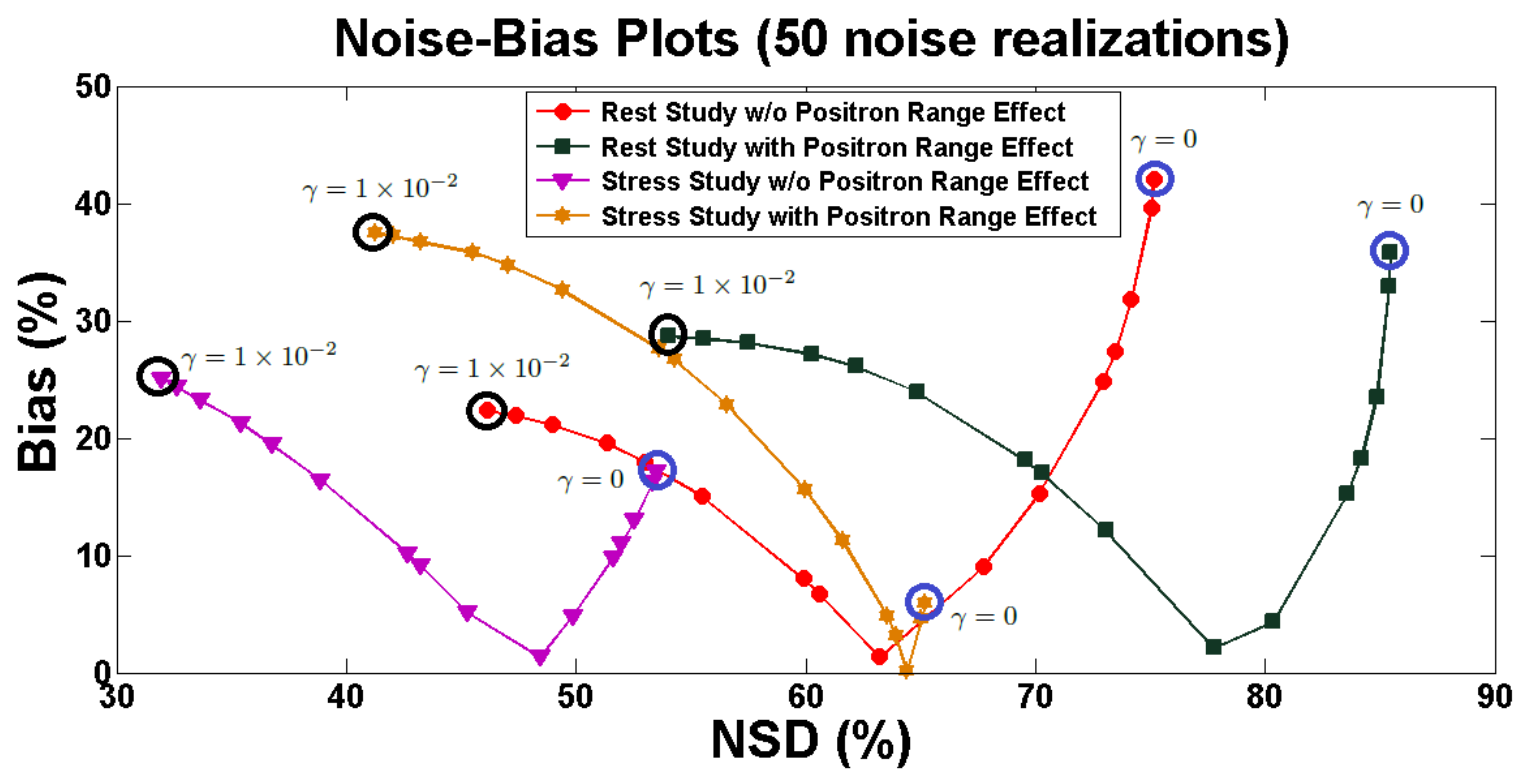

Figure 5. Noise-Bias plots for $K_{1}$ images. Right-most point corresponds to $\gamma=0$ and left-most point corresponds to $\gamma=1 \times 10^{-2}$.

Figure 6 shows MSE of $K_{1}$ parametric images for rest and stress simulations with varying $\gamma$. We observe that for $0<\gamma \leq \gamma^{*}$, MSE decreases monotonically due to reduction in noise and noise-induced bias in the estimation process. $\gamma^{*}$ gives the minimum MSE performance. For $\gamma>\gamma^{*}$, MSE worsens due to substantial increase in bias as compared to modest improvement in noise performance. Without positron range blurring, $K_{1}$ rest images showed a $\sim 98 \%$ reduction in $\operatorname{MSE}\left(\gamma^{*}=6 \times 10^{-4}\right)$, and $K_{1}$ stress images showed a $\sim 96 \%$ reduction in MSE $\left(\gamma^{*}=3 \times 10^{-4}\right)$. With positron range blurring, $K_{1}$ rest images showed a $\sim 98 \%$ reduction in $\operatorname{MSE}\left(\gamma^{*}=6 \times 10^{-4}\right)$, and $K_{1}$ stress images showed a $\sim 5 \%$ reduction in MSE $\left(\gamma^{*}=5 \times 10^{-5}\right)$.

Table 2 shows the mean and standard deviation of flow estimates and CFR in normally perfused myocardium and perfusion defect for rest and stress simulations (without positron range blurring). As $\gamma$ increased, the coefficient of variation $\left(\mathrm{COV}=\frac{\sigma}{\mu}\right)$ for $\mathrm{MBF}_{\text {rest }}$ decreased by $\sim 3 \%$, for $\mathrm{MBF}_{\text {stress }} \mathrm{COV}$ decreased by $\sim 5 \%$, and 


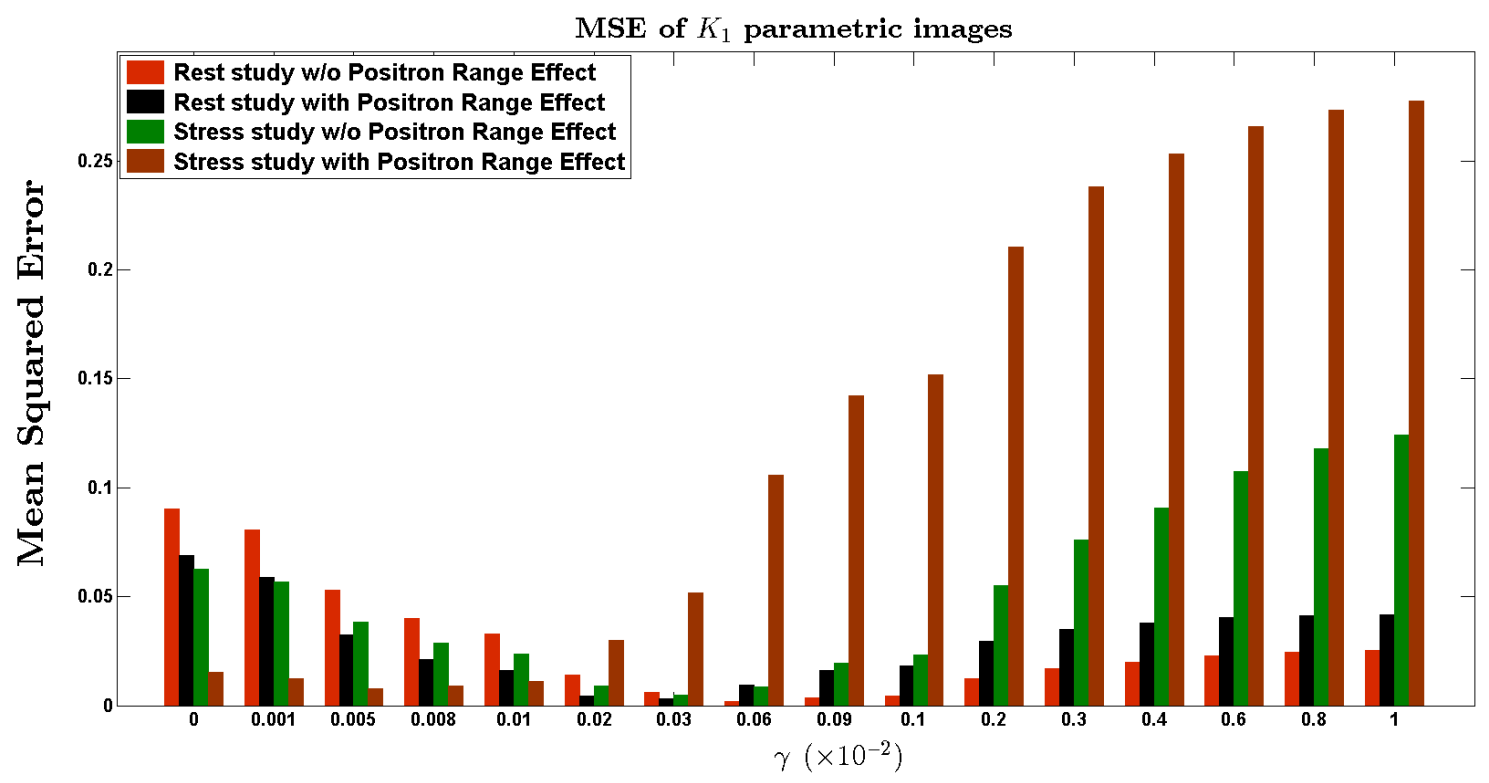

Figure 6. MSE of $K_{1}$ parametric images with varying $\gamma$.

for CFR COV decreased by $\sim 3 \%$. These results further strengthen the observation that physiological clustering produces MBF and CFR estimates with higher SNR by reducing noise and noise-induced bias in the estimation process.

\section{CONCLUSIONS}

Parametric imaging based on physiological clustering clearly outperforms conventional parameter estimation techniques by producing images with higher SNR. It substantially reduces noise and noise-induced bias by utilizing kinetic information from physiologically similar voxels. Functionally similar voxels are binned in the same cluster with each cluster represented by mean kinetics. The mean kinetic information for each cluster is enforced as a spatial constraint in the voxel-wise parameter estimation process thereby forcing (by tuning the penalty parameter $\gamma$ ) the estimated parameters to be close to the mean kinetics of its representative cluster. Unlike previous approaches, it avoids resolution degradation as no spatial smoothing of heterogeneous functional regions is performed. This approach is quite promising in clinical applications as the algorithm is extremely fast and no heavy computations is performed. This work reinforced the need of positron range modeling in the reconstruction process for ${ }^{82} R b$ radio-tracer to obviate resolution degradation due to $\mathrm{PVE}$ and noise propagation in parametric image estimation. Future work involves evaluation on real patient data and extending the concept of physiological clustering in direct 4-D methods. ${ }^{53-59}$

\section{REFERENCES}

[1] Murray, C. J. and Lopez, A. D., "Global mortality, disability, and the contribution of risk factors: Global burden of disease study," The Lancet 349(9063), 1436-1442 (1997).

[2] Gulati, M., Pandey, D. K., Arnsdorf, M. F., Lauderdale, D. S., Thisted, R. A., Wicklund, R. H., Al-Hani, A. J., and Black, H. R., "Exercise capacity and the risk of death in women the st james women take heart project," Circulation 108(13), 1554-1559 (2003).

[3] Thom, T., Haase, N., Rosamond, W., Howard, V. J., Rumsfeld, J., Manolio, T., Zheng, Z.-J., Flegal, K., Odonnell, C., Kittner, S., et al., "Heart disease and stroke statistics2006 update a report from the american heart association statistics committee and stroke statistics subcommittee," Circulation 113(6), e85-e151 (2006).

[4] Mensah, G. A. and Brown, D. W., "An overview of cardiovascular disease burden in the united states," Health Affairs 26(1), 38-48 (2007).

[5] Schindler, T. H., Schelbert, H. R., Quercioli, A., and Dilsizian, V., "Cardiac pet imaging for the detection and monitoring of coronary artery disease and microvascular health," JACC: Cardiovascular Imaging 3(6), 623-640 (2010). 
Table 2. MBF and CFR estimates for rest and stress simulations in normally perfused myocardium and perfusion defects.

\begin{tabular}{|c|c|c|c|c|c|c|}
\hline$\gamma$ & $\mathbf{M B F}_{\text {rest }}^{\text {myo }}$ & $\mathbf{M B F}_{\text {stress }}^{\text {mo }}$ & $\mathbf{M B F}_{\text {rest }}^{\text {defect }}$ & $\mathbf{M B F}_{\text {stress }}^{\text {defect }}$ & $\mathbf{C F R}_{\text {myo }}$ & $\mathbf{C F R}_{\text {defect }}$ \\
\hline 0 & $2.73 \pm 0.29$ & $5.45 \pm 0.34$ & $3.36 \pm 0.76$ & $3.93 \pm 1.01$ & $2.02 \pm 0.24$ & $1.20 \pm 0.33$ \\
\hline $5 \times 10^{-5}$ & $2.45 \pm 0.27$ & $5.22 \pm 0.33$ & $3.14 \pm 0.68$ & $3.69 \pm 0.93$ & $2.15 \pm 0.25$ & $1.21 \pm 0.32$ \\
\hline $8 \times 10^{-5}$ & $2.32 \pm 0.25$ & $5.10 \pm 0.32$ & $3.03 \pm 0.64$ & $3.55 \pm 0.90$ & $2.22 \pm 0.26$ & $1.20 \pm 0.32$ \\
\hline $1 \times 10^{-4}$ & $2.25 \pm 0.24$ & $5.03 \pm 0.32$ & $2.96 \pm 0.62$ & $3.48 \pm 0.88$ & $2.26 \pm 0.26$ & $1.20 \pm 0.32$ \\
\hline $3 \times 10^{-4}$ & $1.82 \pm 0.19$ & $4.53 \pm 0.28$ & $2.52 \pm 0.48$ & $3.00 \pm 0.71$ & $2.52 \pm 0.29$ & $1.22 \pm 0.30$ \\
\hline $6 \times 10^{-4}$ & $1.54 \pm 0.16$ & $4.14 \pm 0.25$ & $2.20 \pm 0.38$ & $2.66 \pm 0.58$ & $2.71 \pm 0.30$ & $1.24 \pm 0.29$ \\
\hline $9 \times 10^{-4}$ & $1.41 \pm 0.14$ & $3.91 \pm 0.23$ & $2.02 \pm 0.33$ & $2.47 \pm 0.51$ & $2.80 \pm 0.31$ & $1.25 \pm 0.29$ \\
\hline $1 \times 10^{-3}$ & $1.37 \pm 0.14$ & $3.85 \pm 0.23$ & $1.97 \pm 0.32$ & $2.42 \pm 0.49$ & $2.82 \pm 0.31$ & $1.25 \pm 0.29$ \\
\hline $3 \times 10^{-3}$ & $1.12 \pm 0.11$ & $3.29 \pm 0.21$ & $1.60 \pm 0.24$ & $2.01 \pm 0.37$ & $2.96 \pm 0.34$ & $1.29 \pm 0.32$ \\
\hline $8 \times 10^{-3}$ & $1.01 \pm 0.10$ & $2.98 \pm 0.21$ & $1.41 \pm 0.21$ & $1.82 \pm 0.34$ & $2.97 \pm 0.36$ & $1.33 \pm 0.35$ \\
\hline $1 \times 10^{-2}$ & $1.00 \pm 0.10$ & $2.94 \pm 0.20$ & $1.38 \pm 0.21$ & $1.80 \pm 0.34$ & $2.97 \pm 0.37$ & $1.34 \pm 0.36$ \\
\hline True Values & $\mathbf{1 . 3 6}$ & $\mathbf{4 . 4 8}$ & $\mathbf{1 . 3 6}$ & $\mathbf{1 . 5 0}$ & $\mathbf{3 . 3 0}$ & $\mathbf{1 . 1 1}$ \\
\hline
\end{tabular}

[6] Organization, W. H., [World health statistics 2011], World Health Organization (2011).

[7] Dilsizian, V. and Narula, J., [Atlas of nuclear cardiology], Springer (2013).

[8] Bengel, F. M., "Methodology for quantifying absolute myocardial perfusion with pet and spect," Current cardiology reports $\mathbf{9}(2), 121-128$ (2007).

[9] Burckhardt, D. D., Declerck, J., and Pan, X.-B., "Cardiac positron emission tomography: Overview of myocardial perfusion, myocardial blood flow and coronary flow reserve imaging," www.siemens.com/mi , 1-12 (2009).

[10] Uren, N. G., Melin, J. A., De Bruyne, B., Wijns, W., Baudhuin, T., and Camici, P. G., "Relation between myocardial blood flow and the severity of coronary-artery stenosis," New England Journal of Medicine 330(25), 1782-1788 (1994).

[11] Parkash, R., Dekemp, R., Ruddy, T., Kitsikis, A., Hart, R., Beauschene, L., Williams, K., Davies, R., Labinaz, M., and Beanlands, R., "Potential utility of rubidium 82 pet quantification in patients with 3-vessel coronary artery disease," Journal of nuclear cardiology 11(4), 440-449 (2004).

[12] Yoshida, K., Mullani, N., and Gould, K. L., "Coronary flow and flow reserve by pet simplified for clinical applications using rubidium-82 or nitrogen-13-ammonia," Journal of Nuclear Medicine 37(10), 1701-1712 (1996).

[13] Bengel, F. M., Higuchi, T., Javadi, M. S., and Lautamaki, R., "Cardiac positron emission tomography," Journal of the American College of Cardiology 54(1), 1-15 (2009).

[14] Nakazato, R., Berman, D. S., Alexanderson, E., and Slomka, P., "Myocardial perfusion imaging with pet," Imaging 5(1), 35-46 (2013).

[15] Lodge, M. A., Braess, H., Mahmoud, F., Suh, J., Englar, N., Geyser-Stoops, S., Jenkins, J., Bacharach, S. L., Dilsizian, V., et al., "Developments in nuclear cardiology: Transition from single photon emission computed tomography to positron emission tomography/computed tomography," JOURNAL OF INVASIVE CARDIOLOGY 17(9), 491 (2005).

[16] Rahmim, A. and Zaidi, H., "Pet versus spect: strengths, limitations and challenges," Nuclear medicine communications 29(3), 193-207 (2008).

[17] Salerno, M. and Beller, G. A., "Noninvasive assessment of myocardial perfusion," Circulation: Cardiovascular Imaging 2(5), 412-424 (2009).

[18] O Donnell, J. K., Wojtylak, P., and Faulhaber, P. F., "Myocardial perfusion imaging: past, present and future," MedicaMundi 54/1, 46-49 (2010).

[19] Sciagra, R., "Quantitative cardiac positron emission tomography: The time is coming!," Scientifica 2012 (2012).

[20] Yoshinaga, K., Klein, R., and Tamaki, N., "Generator-produced rubidium-82 positron emission tomography myocardial perfusion imagingfrom basic aspects to clinical applications," Journal of cardiology 55(2), 163-173 (2010).

[21] Arumugam, P., Tout, D., and Tonge, C., "Myocardial perfusion scintigraphy using rubidium-82 positron emission tomography," British medical bulletin 107(1), 87-100 (2013).

[22] Dilsizian, V., "Spect and pet myocardial perfusion imaging: Tracers and techniques," in [Atlas of Nuclear Cardiology], 55-93, Springer (2013).

[23] El Fakhri, G., Sitek, A., Guérin, B., Kijewski, M. F., Di Carli, M. F., and Moore, S. C., "Quantitative dynamic cardiac 82rb pet using generalized factor and compartment analyses," Journal of Nuclear Medicine 46(8), 1264-1271 (2005).

[24] Klein, R., Bentourkia, M., Beanlands, R. S., Adler, A., and deKemp, R., "A minimal factor overlap method for resolving ambiguity in factor analysis of dynamic cardiac pet," in [Nuclear Science Symposium Conference Record, 200\%. NSS'0\%. IEEE], 5, 3268-3272, IEEE (2007). 
[25] Keiichiro Yoshinaga, M. and deKemp PhD, R. A., "Quantification of regional myocardial blood flow estimation with three-dimensional dynamic rubidium-82 pet and modified spillover correction model," Journal of Nuclear Cardiology 19(4), 763-774 (2012).

[26] Lortie, M., Beanlands, R. S., Yoshinaga, K., Klein, R., DaSilva, J. N., et al., "Quantification of myocardial blood flow with 82rb dynamic pet imaging," European journal of nuclear medicine and molecular imaging 34(11), 1765-1774 (2007).

[27] Klein, R., Beanlands, R. S., and deKemp PhD, R. A., "Quantification of myocardial blood flow and flow reserve: Technical aspects," Journal of nuclear cardiology 17(4), 555-570 (2010).

[28] Klein, R., Kinetic Model Based Factor Analysis of Cardiac 82 Rb PET Images for Improved Accuracy of Quantitative Myocardial Blood Flow Measurement, PhD thesis, University of Ottawa (2010).

[29] Beanlands, R. S. and deKemp PhD, R. A., "Characterizing the normal range of myocardial blood flow with 82rubidium and 13n-ammonia pet imaging," Journal of Nuclear Cardiology, 1-14 (2013).

[30] Renkin, E. M., "Transport of potassium-42 from blood to tissue in isolated mammalian skeletal muscles," American Journal of Physiology-Legacy Content 197(6), 1205-1210 (1959).

[31] Crone, C., "The permeability of capillaries in various organs as determined by use of the indicator diffusionmethod," Acta physiologica scandinavica 58(4), 292-305 (1963).

[32] Zanotti-Fregonara, P., Chen, K., Liow, J.-S., Fujita, M., and Innis, R. B., "Image-derived input function for brain pet studies: many challenges and few opportunities," Journal of Cerebral Blood Flow E Metabolism 31(10), 1986-1998 (2011).

[33] Choi, Y., Huang, S.-C., Hawkins, R. A., Kim, J. Y., Kim, B.-T., Hoh, C. K., Chen, K., Phelps, M. E., and Schelbert, H. R., "Quantification of myocardial blood flow using 13n-ammonia and pet: comparison of tracer models.," Journal of nuclear medicine: official publication, Society of Nuclear Medicine 40(6), 1045-1055 (1999).

[34] Wu, H.-M., Hoh, C. K., Choi, Y., Schelbert, H. R., Hawkins, R. A., Phelps, M. E., and Huang, S.-C., "Factor analysis for extraction of blood time-activity curves in dynamic fdg-pet studies," The Journal of nuclear medicine 36(9), 1714-1722 (1995).

[35] Ahn, J.-H., Kim, S., Oh, J.-H., and Choi, S., "Multiple nonnegative-matrix factorization of dynamic pet images," in [Proceedings of Asian Conference on Computer Vision], (2004).

[36] Schulz, D., Tapfer, A., Buck, A., Reeder, S., Miederer, M., et al., "Non-negative matrix factorization based input function extraction for mouse imaging in small animal pet-comparison with arterial blood sampling and factor analysis," J Mol Imaging Dynam 2(108), 2 (2012).

[37] Samal, M., Karny, M., Surova, H., Penicka, P., Marikova, E., and Dienstbier, Z., "On the existence of an unambiguous solution in factor analysis of dynamic studies," Physics in medicine and biology 34(2), 223 (1989).

[38] Donoho, D. and Stodden, V., "When does non-negative matrix factorization give a correct decomposition into parts?," in [Advances in neural information processing systems], None (2003).

[39] Klein, R., Beanlands, R., Wassenaar, R., Thorn, S., Lamoureux, M., DaSilva, J., Adler, A., et al., "Kinetic model-based factor analysis of dynamic sequences for 82-rubidium cardiac positron emission tomography," Medical physics 37, 3995 (2010).

[40] Lee, J. S., Lee, D. D., Choi, S., Park, K. S., and Lee, D. S., "Non-negative matrix factorization of dynamic images in nuclear medicine," in [Nuclear Science Symposium Conference Record, 2001 IEEE], 4, 2027-2030, IEEE (2001).

[41] Kimura, Y., Hsu, H., Toyama, H., Senda, M., and Alpert, N. M., "Improved signal-to-noise ratio in parametric images by cluster analysis," Neuroimage 9(5), 554-561 (1999).

[42] Wong, K.-P., Feng, D., Meikle, S. R., and Fulham, M. J., "Segmentation of dynamic pet images using cluster analysis," Nuclear Science, IEEE Transactions on 49(1), 200-207 (2002).

[43] Zhou, Y., Huang, S.-C., Bergsneider, M., and Wong, D. F., "Improved parametric image generation using spatialtemporal analysis of dynamic pet studies," NeuroImage 15(3), 697-707 (2002).

[44] Guo, H., Renaut, R., Chen, K., and Reiman, E., "Clustering huge data sets for parametric pet imaging," Biosystems 71(1), 81-92 (2003).

[45] Huang, X., Zhou, Y., Bao, S., and Huang, S.-C., "Clustering-based linear least square fitting method for generation of parametric images in dynamic fdg pet studies," Journal of Biomedical Imaging 2007(2), 3 (2007).

[46] Saad, A., Smith, B., Hamarneh, G., and Möller, T., "Simultaneous segmentation, kinetic parameter estimation, and uncertainty visualization of dynamic pet images," in [Medical Image Computing and Computer-Assisted InterventionMICCAI 2007], 726-733, Springer (2007).

[47] Chi, E. C. and Lange, K., "Splitting methods for convex clustering," arXiv preprint arXiv:1304.0499 (2013).

[48] Tatiraju, S. and Mehta, A., "Image segmentation using k-means clustering, em and normalized cuts," University Of California Irvine (2008).

[49] Mouysset, S., Zbib, H., Stute, S., Girault, J., Charara, J., Noailles, J., Chalon, S., Buvat, I., and Tauber, C., "Segmentation of dynamic pet images with kinetic spectral clustering," Physics in medicine and biology 58(19), 6931 (2013). 
[50] Zbib, H., Mouysset, S., Stute, S., Girault, J.-M., Charara, J., Chalon, S., Galineau, L., Buvat, I., and Tauber, C., "Optimized spectral clustering for segmentation of dynamic pet images," in [Advances in Biomedical Engineering (ICABME), 2013 2nd International Conference on], 22-25, IEEE (2013).

[51] Leader, J. J., [Numerical analysis and scientific computation], Pearson Addison Wesley Boston (2004).

[52] Rahmim, A., Tang, J., Lodge, M., Lashkari, S., Ay, M. R., Lautamäki, R., Tsui, B., and Bengel, F., "Analytic system matrix resolution modeling in pet: an application to rb-82 cardiac imaging," Physics in medicine and biology $\mathbf{5 3}(21)$, 5947 (2008).

[53] Kamasak, M. E., Bouman, C. A., Morris, E. D., and Sauer, K., "Direct reconstruction of kinetic parameter images from dynamic pet data," Medical Imaging, IEEE Transactions on 24(5), 636-650 (2005).

[54] Tsoumpas, C., Turkheimer, F. E., and Thielemans, K., "A survey of approaches for direct parametric image reconstruction in emission tomography," Medical physics 35, 3963 (2008).

[55] Rahmim, A., Tang, J., and Zaidi, H., "Four-dimensional (4d) image reconstruction strategies in dynamic pet: beyond conventional independent frame reconstruction," Medical physics 36, 3654 (2009).

[56] Wang, G. and Qi, J., "Direct estimation of kinetic parametric images for dynamic pet," Theranostics 3(10), 802-815 (2013).

[57] Rakvongthai, Y., Ouyang, J., Guerin, B., Li, Q., Alpert, N. M., and El Fakhri, G., "Direct reconstruction of cardiac pet kinetic parametric images using a preconditioned conjugate gradient approach," Medical physics 40(10), 102501 (2013).

[58] Su, K.-H., Yen, T.-C., and Fang, Y.-H. D., "A novel approach for direct reconstruction of parametric images for myocardial blood flow from pet imaging," Medical physics 40(10), 102505 (2013).

[59] Rahmim, A., Tang, J., and Mohy-ud Din, H., "Direct 4d parametric imaging in dynamic myocardial perfusion pet," Frontiers in Biomed. Tech. 1, 4-13 (2014). 